\title{
Antitumor activity of combination treatment with gefitinib and docetaxel in EGFR-TKI-sensitive, primary resistant and acquired resistant human non-small cell lung cancer cells
}

\author{
MIN WU ${ }^{1}$, YUAN YUAN ${ }^{2}$, YUE-YIN PAN $^{3}$ and YING ZHANG ${ }^{1}$ \\ ${ }^{1}$ Department of Geriatrics, ${ }^{2}$ The Central Laboratory of Binhu Hospital, \\ The Third Affiliated Hospital of Anhui Medical University, Hefei, Anhui 230061; \\ ${ }^{3}$ Department of Oncology, The First Affiliated Hospital of Anhui Medical University, \\ Hefei, Anhui 230022, P.R. China
}

Received August 4, 2013; Accepted February 24, 2014

DOI: $10.3892 / \mathrm{mmr} .2014 .2082$

\begin{abstract}
In a number of large clinical studies, concurrent administration of the epidermal growth factor receptor-tyrosine kinase inhibitor (EGFR-TKI) with cytotoxic chemotherapy has failed to improve the survival rate in unselected patients with advanced non-small cell lung cancer (NSCLC). The purpose of the current study was to investigate the antitumor effects of gefitinib in combination with docetaxel in EGFR-TKI-sensitive, primary resistant and acquired resistant human lung cancer cell lines and the associated molecular mechanisms. EGFR-TKI-sensitive and EGFR-TKI-resistant human lung cancer cell lines were exposed to gefitinib or docetaxel alone, or in combination. Cell viability was assessed using the MTT assay. Cell cycle distribution and apoptosis were measured by flow cytometry and alterations in signaling pathways were examined by immunoblotting. The cytotoxic interaction between docetaxel and gefitinib was determined by combination index (CI) analysis. Coadministration of gefitinib and docetaxel was observed to result in superior inhibition of tumor cell proliferation, however, increased rates of apoptosis were only observed in EGFR-TKI-sensitive cells, whereas, antagonistic activity was observed in the EGFR-TKI-resistant cell lines. Gefitinib arrested the cell cycle at the G1 phase, whereas docetaxel arrested the cell cycle at the $\mathrm{S}$ phase. In addition, in cells exhibiting a synergistic interaction between gefitinib and docetaxel, an increase in p-EGFR and
\end{abstract}

Correspondence to: Professor Yue-Yin Pan, Department of Oncology, The First Affiliated Hospital of Anhui Medical University, 218 Ji Xi Road, Hefei, Anhui 230022, P.R. China

E-mail: panyueyin1965@126.com

Professor Ying Zhang, Department of Geriatrics, The Third Affiliated Hospital of Anhui Medical University, 390 Huai He Road, Hefei, Anhui 230061, P.R. China

E-mail: yingzhang1965@163.com

Key words: non-small-cell lung cancer, combined modality therapy, gefitinib, docetaxel
p-AKT was observed following chemotherapy exposure. By contrast, in cells exhibiting no change or a decrease in p-EGFR and p-AKT following docetaxel treatment, an antagonistic interaction between the two agents was observed. In conclusion, the combination of docetaxel and gefitinib generated synergistic effects in EGFR-TKI-sensitive cells and antagonistic effects in EGFR-TKI-primary and acquired resistant cells, suggesting that EGFR-TKIs, combined with docetaxel, may be beneficial to NSCLC patients with EGFR mutations. The results also indicate that the interactions between gefitinib and docetaxel may be associated with the effect of docetaxel on EGFR phosphorylation.

\section{Introduction}

Non-small cell lung cancer (NSCLC) is the most common type of lung cancer accounting for $80-85 \%$ of all lung cancer cases (1). At present, traditional cytotoxic chemotherapy has reached a therapeutic plateau with limited survival benefits for advanced NSCLC patients and novel combinations of available cytotoxic agents are unlikely to confer clinically relevant survival improvement (2).

Treatment with targeted agents has improved progression-free and overall survival in patients with a variety of tumors, including NSCLC. Gefitinib and erlotinib, as certain epidermal growth factor receptor tyrosine kinase inhibitors (EGFR-TKIs) have been approved as second-line treatments for lung cancer (3).

Marked response rates of EGFR-TKIs are associated with activating mutations in the EGFR gene (4). Despite this patients with K-ras mutations have been shown to confer primary resistance to gefitinib and erlotinib therapy (5). In addition, patients with EGFR-mutant tumors who initially respond to treatment with EGFR-TKIs are likely to develop progressive disease following one year of EGFR-TKI treatment, a hypothesis referred to clinically as acquired resistance (6). Regardless of considerable efforts for the improvement in diagnosis and treatment of lung cancer, the majority of patients present at an advanced stage and prognosis remains poor, with an overall 5 -year survival probability of $\sim 15 \%(7,8)$. Therefore, there is a 
requirement to identify novel treatment strategies to improve the outcomes of patients with lung cancer.

Docetaxel is a well-established anticancer agent and a member of the taxoid family. It is a mitotic inhibitor that promotes the assembly of microtubules from tubulin dimers and stabilizes microtubules by preventing depolymerization (9). In this manner, docetaxel specifically arrests the cell cycle at the $\mathrm{S}$ or G2/M phase and induces apoptosis in tumor cells (10).

At present, there is interest in assessing the efficacy of EGFR-TKIs administered in combination with cytotoxic chemotherapeutic agents. Gefitinib is the first EGFR tyrosine kinase inhibitor that has been shown to be an effective monotherapy for the treatment of chemotherapy-failed advanced non-small cell lung cancer $(11,12)$. Preclinical studies suggested that the combination of gefitinib with chemotherapy was expected to improve survival $(13,14)$. However, despite preclinical data suggesting additive or synergistic effects when combining EGFR-TKIs with chemotherapy, concurrent administration of the EGFR-TKI gefitinib with first-line chemotherapies has failed to improve survival in unselected patients with advanced NSCLC in two large clinical trial studies $(15,16)$. Two hypotheses have been proposed to explain the unexpected negative outcomes of the EGFR-TKIs and chemotherapy combination studies in advanced-stage NSCLC: i) Lack of patient selection with a predictive marker; and ii) incorrect choice of chemotherapeutic agent, dose and regimen $(17,18)$. Thus, one of the major challenges for an optimal use of combination EGFR-TKIs and chemotherapy is to determine which patients are more likely to gain a therapeutic advantage from the treatment.

In the present study, EGFR-TKI-sensitive, primary resistant and acquired resistant human lung cancer cell lines were used as in vitro models to define the differential effects of gefitinib and docetaxel combination on cell proliferation, apoptosis, cell cycle distribution and signaling pathways.

\section{Materials and methods}

Drugs. Docetaxel (Taxotere; Sanofi Aventis, Labège, France) was purchased as a commercial product from our hospital pharmacy and was dissolved in dimethylsulfoxide (DMSO) at $1 \mathrm{mM}$, as a stock solution. Gefitinib (Iressa) was obtained from AstraZeneca (London, UK) and was dissolved in DMSO to a stock concentration of $10 \mathrm{mM}$. The drugs were stored at $-20^{\circ} \mathrm{C}$ and diluted with culture medium prior to use. The final concentration of DMSO in the Dulbecco's modified Eagle's medium (DMEM) was maintained at $<0.1 \%$.

Cell lines. The EGFR-TKI primary resistant A549 lung cancer cell line (mutant KRAS/wild-type EGFR) was purchased from the American Type Culture Collection (Manassas, VA, USA). The EGFR-TKI-sensitive PC9 (mutant EGFR/wild-type K-Ras) and the gefitinib-acquired-resistant PC9/GR human NSCLC cell lines were provided by Dr Xuchao Zhang, Guangdong Lung Cancer Institute (Guangdong, China). A549, PC9 and PC9/GR cell lines were cultured in DMEM (HyClone, Logan, UT, USA) supplemented with $10 \%$ fetal bovine serum, penicillin $(100 \mathrm{UI} / \mathrm{ml})$ and streptomycin $(100 \mu \mathrm{g} / \mathrm{ml})$ at $37^{\circ} \mathrm{C}$ in a humidified atmosphere with $5 \% \mathrm{CO}_{2}$, and harvested with trypsin-EDTA when the cells had reached exponential growth.
Cell proliferation assay. The MTT assay was used to determine the antitumor effects of each drug. In brief, cells were plated in 96-well plates, in which the number of cells per well was 3,000 A549 cells, 3,500 PC-9 cells and 3,500 PC9/GR cells. Following overnight culture, cells were treated with increasing doses of gefitinib or docetaxel for $72 \mathrm{~h}$. The $\mathrm{IC}_{50}$ value was the concentration resulting in $50 \%$ cell growth inhibition by a $72 \mathrm{~h}$ exposure to drug compared with the untreated control cells. Following cell exposure to each drug for $72 \mathrm{~h}$ in 96-well plates, $20 \mu \mathrm{l} \mathrm{MTT}$ $(5 \mathrm{mg} / \mathrm{ml})$ solution was added to each well and then the optical density (OD) of each well was determined at $490 \mathrm{~nm}$ on an ELISA plate reader (Bio-Rad Laboratories, Inc., Winooski, VT, USA) following $4 \mathrm{~h}$ incubation at $37^{\circ} \mathrm{C}$. The percentage of cell growth inhibition resulting from each drug was calculated as: $\left[\left(\mathrm{OD} 490_{\text {control cells }}-\mathrm{OD} 490_{\text {treated cells }}\right) / \mathrm{OD} 490_{\text {control cells }}\right] \times 100$. This assay was repeated in more than three independent experiments.

Analysis of interactions. To evaluate the antiproliferative effects of the combined treatment, the A549, PC9 and PC9/GR cells were concurrently exposed to gefitinib and docetaxel for $72 \mathrm{~h}$. The two drugs were combined in a constant ratio of doses that typically corresponded to $0.125,0.25,0.5,1,2$ and 4 times that of the individual $\mathrm{IC}_{50} \mathrm{~s}$. Interactions between gefitinib and docetaxel were expressed as the combination index (CI) according to the Chou and Talaly method using CalcuSyn software (ComboSyn, Inc., Paramus, NJ, USA): $\mathrm{CI}>1, \mathrm{CI}=1$ and $\mathrm{CI}<1$ indicate antagonistic, additive and synergistic effects, respectively (19).

Cell cycle analysis. Cell cycle analysis was conducted using flow cytometry. Cells (1x10\%/well) were seeded into six-well plates. Following $24 \mathrm{~h}$ incubation, the cells were treated with docetaxel and gefitinib as single agents or in combination at the concentration of $\mathrm{IC}_{50}$ levels for $72 \mathrm{~h}$. Next, the adhered cells were harvested by trypsinization, washed twice with phosphate-buffered saline (PBS) and fixed in $75 \%$ cold ethanol at $4{ }^{\circ} \mathrm{C}$ overnight. DNA staining was performed using a solution with propidium iodide (PI; $50 \mu \mathrm{g} / \mathrm{ml}), 0.1 \%$ Triton $\mathrm{X}-100$ and RNase $(200 \mu \mathrm{g} / \mathrm{ml})$ in the dark for $30 \mathrm{~min}$ at room temperature. Cells were analyzed using a FACScan cytometer (Becton Dickinson, San Jose, CA, USA) and the percentages of cells in the G0/G1, S and G2/M phases of the cell cycle were estimated using ModFit LT 4.0 software (Verity Software House, Topsham, ME, USA).

Annexin $V$ assay for assessment of apoptosis. The effects of each individual and combination of drugs on apoptosis were analyzed in A549, PC9 and PC9/GR cell lines, using flow cytometry. As a standard, $2 \times 10^{5}$ cells in the exponential growth phase were seeded in $60 \mathrm{~mm}^{2}$ dishes. After $24 \mathrm{~h}$ incubation, cells were treated with single or double drugs using the $\mathrm{IC}_{50}$ concentration, as for the growth inhibition assay. After $72 \mathrm{~h}$ treatment, the adherent and floating cells were collected, washed twice with PBS, resuspended in an Annexin V binding buffer, and incubated with $5 \mu \mathrm{l}$ Annexin $\mathrm{V}$ and $10 \mu \mathrm{l}$ propidium iodide $(40 \mu \mathrm{g} / \mathrm{ml})$ at room temperature in the dark for $15 \mathrm{~min}$. Following incubation, the stained cells were analyzed using a flow cytometer. Cells with no drug treatment were used as a control. Data was analyzed by CellQuest software (Becton Dickinson). 
A

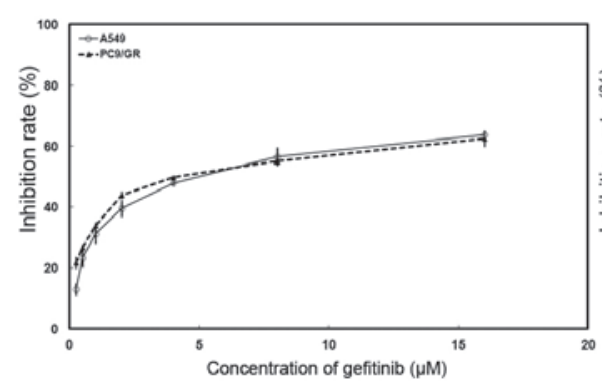

B

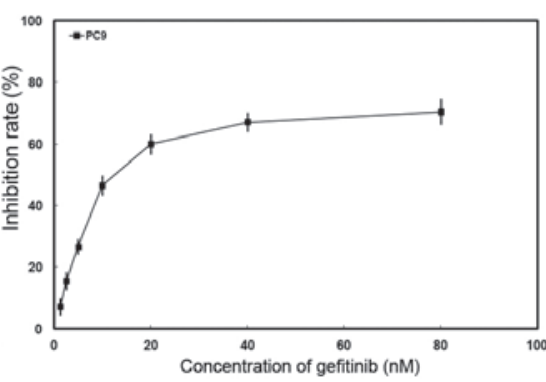

C

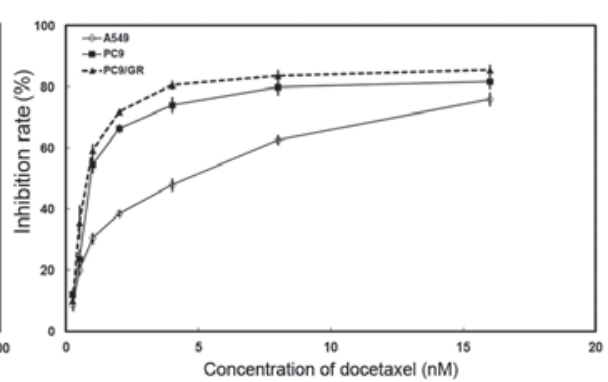

Figure 1. MTT assays were used to examine the inhibitory activity of gefitinib and docetaxel on cell proliferation. The cells were exposed to varying concentrations of gefitinib in (A) A549 and PC9/GR cells (0.25-16 $\mu \mathrm{mol} / \mathrm{l})$ and (B) PC9 cells (1.25-80 nmol/1) or (C) docetaxel (0.25-16 nmol/1) in all three cells for 72 h. Each data point is the result of more than three independent experiments.

Western blot analysis. A549, PC9 and PC9/GR cells (1x10/well) were cultured on $100 \mathrm{~mm}^{2}$ plates overnight and treated with gefitinib and docetaxel as single agents and in combination for $72 \mathrm{~h}$, at $\mathrm{IC}_{50}$ levels. The cells were washed with ice-cold PBS solution and scraped in lysis buffer. The lysates were centrifuged at $13,380 \mathrm{x}$ for $30 \mathrm{~min}$ at $4^{\circ} \mathrm{C}$ and the supernatant was collected. Equivalent cellular proteins were analyzed by sodium dodecyl sulfate-polyacrylamide gel electrophoresis (SDS-PAGE) and transferred to polyvinylidine difluoride (PVDF) membranes. The membranes were blocked in PBS buffer containing 5\% milk and $0.1 \%$ Tween- 20 for $2 \mathrm{~h}$. Next, the appropriate primary antibodies against pY1068 EGFR, EGFR, ps473AKT, AKT and $\beta$-actin purchased from Cell Signaling Technology (Beverly, MA, USA) were used and incubated overnight at $4{ }^{\circ} \mathrm{C}$. Visualized of proteins was performed with a horseradish peroxidasecoupled secondary antibody from Cell Signaling Technology at room temperature for $1 \mathrm{~h}$. Specific bands were detected using the enhanced chemiluminescence reagents (Millipore, Billerica, MA, USA). Equal loading was assessed by immunoblotting for $\beta$-actin, total EGFR or total AKT, as indicated.

Statistical analysis. The results obtained from at least three independent experiments are expressed as the mean \pm standard deviation. Student's t-test and one-way ANOVA used to determine the differences between control and treatment groups. $\mathrm{P}<0.05$ was considered to indicate a statistically significant result.

\section{Results}

Different antiproliferative effects of gefitinib and docetaxel in EGFR-TKI-sensitive and EGFR-TKI-resistant NSCLC cell lines. The effects of gefitinib and docetaxel on the proliferation of the three NSCLC cells were determined using an MTT assay. Dose-dependent growth inhibitory effects of gefitinib or docetaxel were observed in NSCLC cell lines (Fig. 1). Table I summarizes the $\mathrm{IC}_{50}$ of these two drugs. To investigate the effects of combined treatment, PC9, A549 and PC9/GR cells were exposed to various concentrations of gefitinib and docetaxel concomitantly for $72 \mathrm{~h}$. In EGFR-TKI-sensitive PC9 cells concurrent administration of the drugs resulted in synergistic effects $(\mathrm{CI}<1$; Fig. 2). By contrast, antagonistic activity was observed in EGFR-TKI primary resistant A549 cells and acquired resistant PC9/GR cell lines (CI>1).
Table I. $\mathrm{IC}_{50}$ values of gefitinib and docetaxel were determined by MTT.

\begin{tabular}{lccc}
\hline $\mathrm{IC}_{50}$ & $\mathrm{~A} 549$ & PC9 & PC9/GR \\
\hline Docetaxel & $3.76 \pm 0.32 \mathrm{nM}$ & $1.41 \pm 0.18 \mathrm{nM}$ & $1.05 \pm 0.14 \mathrm{nM}$ \\
Gefitinib & $4.92 \pm 0.79 \mu \mathrm{M}$ & $17.16 \pm 2.62 \mathrm{nM}$ & $4.55 \pm 0.54 \mu \mathrm{M}$ \\
\hline
\end{tabular}

$\mathrm{IC}_{50}$, concentration resulting in inhibition of $50 \%$ of the maximal cell growth.

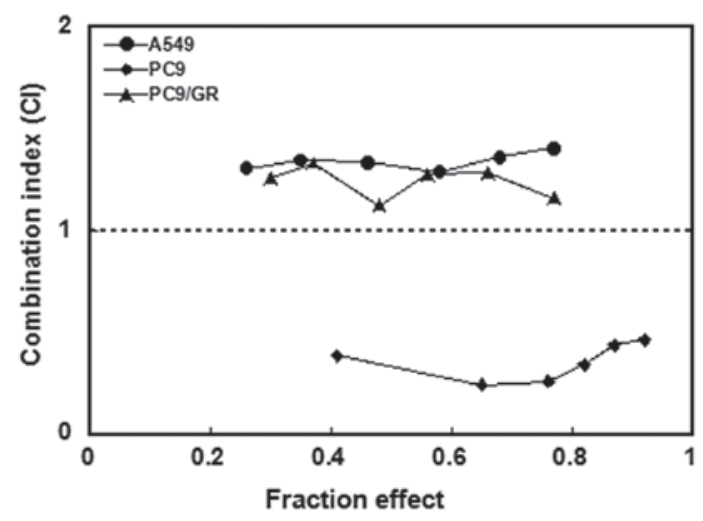

Figure 2. The CI value of gefitinib combined with docetaxel in A549, PC9 and $\mathrm{PC}$ /GR cells was calculated using the Chou-Talalay method. $\mathrm{CI}<1, \mathrm{CI}=1$ and $\mathrm{CI}>1$ indicate synergistic, additive and antagonistic effects, respectively. CI, combination index.

Cell cycle effects of gefitinib and docetaxel. Flow cytometry was used to evaluate the cell cycle phase distribution in the NSCLC cells following single-drug and concurrent administration of gefitinib and docetaxel for $72 \mathrm{~h}$. Cell cycle analysis in the PC9 cell line demonstrated that treatment with gefitinib alone increased the population of cells in the G0/G1 phase with a concomitant decrease in $\mathrm{S}$ phase $(\mathrm{P}<0.05$; Fig. 3$)$. However, in A549 and PC9/GR cell lines, when treated with gefitinib alone marked additional G0/G1 phase arrest was observed. Following administration of docetaxel alone, the $\mathrm{S}$ or G2 phase fraction significantly increased in PC9, A549 and PC9/GR cell lines $(\mathrm{P}<0.05)$. When EGFR-TKI-sensitive PC9 cells were exposed to docetaxel combined with gefitinib, a similar cell cycle arrest pattern (mainly $\mathrm{S}$ phase arrest) as 

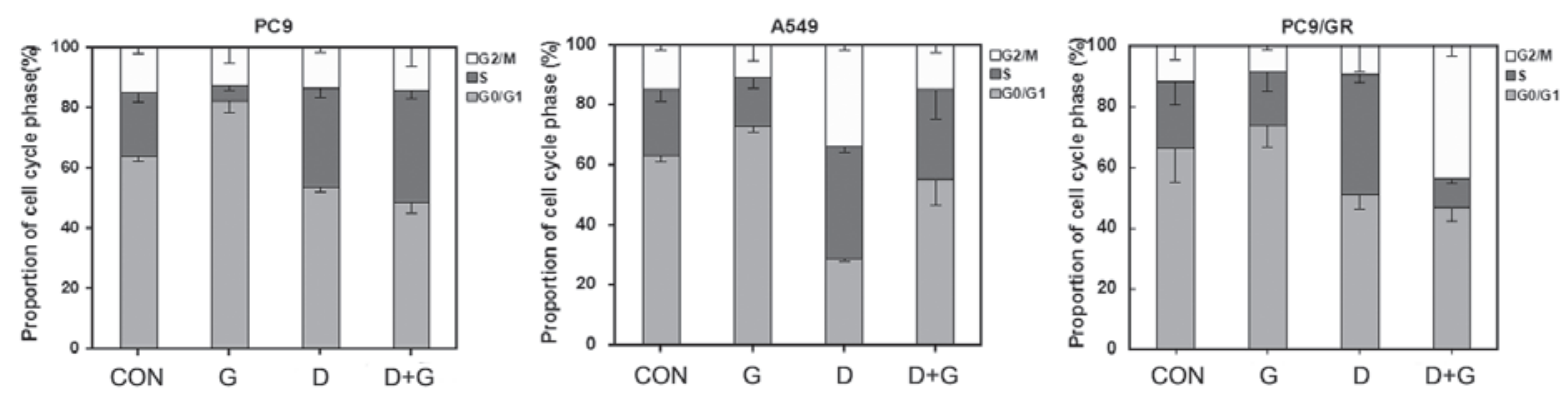

Figure 3. Flow cytometric analysis was used to determine the alterations in the cell cycle distributions in non-small-cell lung cancer cell lines following the administration of gefitinib and docetaxel as single-agents or concurrently for $72 \mathrm{~h}$. The concentrations of docetaxel and gefitinib were used at $\mathrm{IC}_{50}$ levels. CON, G, D and D+G refer to control, gefitinib, docetaxel and concurrent administration, respectively.
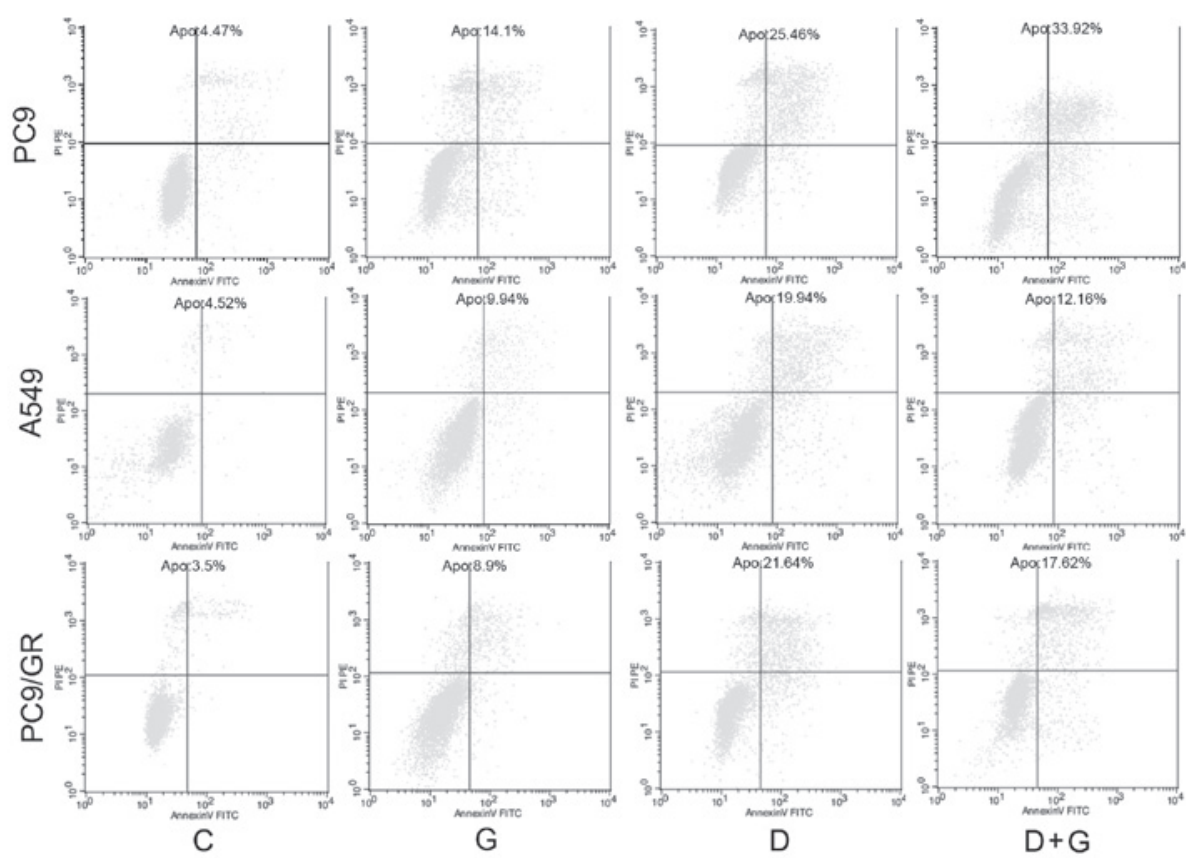

Figure 4. Effect of gefitinib and docetaxel on cell apoptosis. The effects of each single drug and the combination on apoptosis were analyzed in PC9, A549 and PC9/GR cell lines using flow cytometry. Following $72 \mathrm{~h}$ treatment, adherent and floating cells were collected and incubated with Annexin V and PI (x-axis, Annexin V; y-axis, PI). The apoptotic rate was determined using the CellQuest software. PI, propidium iodide. CON, G, D and D+G refer to control, gefitinib, docetaxel and concurrent administration, respectively.

observed with docetaxel administered alone and a corresponding reduction of arrest at the $\mathrm{G} 0 / \mathrm{G} 1$ phase $(\mathrm{P}<0.05)$ was observed. However, in EGFR-TKI-resistant A549 and PC9/GR cells the concurrent administration of docetaxel and gefitinib resulted in alterations in the cell cycle phase distributions and overlapping effects from the two agents.

Effects of docetaxel or gefitinib alone, or in combination on cell apoptosis. To further evaluate whether observed growth inhibition is due to enhanced apoptosis, cell apoptosis analyses were performed using an Annexin V/PI assay. In EGFR-sensitive PC9 cell lines, combined treatment with gefitinib and docetaxel resulted in a significant increase in the apoptotic population compared with cells treated with each single agent (Fig. 4). By contrast, when EGFR-TKI-resistant A549 and PC9/GR cells were exposed to cotreatment with docetaxel and gefitinib, a decrease in apoptosis was observed compared with cells treated with docetaxel alone.
Collectively, these results indicate that gefitinib antagonizes docetaxel-induced apoptosis in NSCLC cell lines with primary and acquired resistance to EGFR-TKI, and has the opposite effect on the EGFR-TKI-sensitive cell line.

Effect of gefitinib or docetaxel, or their combination on EGFR-mediated signaling pathways. To determine the effects of single or combined drugs on the EGFR signaling pathway, the levels of phosphorylated EGFR and phosphorylated AKT in PC9, A549 and PC9/GR cell lines were analyzed by immunoblotting. Cells were exposed to the $\mathrm{IC}_{50}$ concentration of each drug for $72 \mathrm{~h}$. Gefitinib inhibited the activation of EGFR and its downstream signaling mediator, AKT, effectively in gefitinib-sensitive PC9 cells, whereas the inhibitory effects on these signaling pathways in EGFR-TKI-resistant A549 and PC9/GR cells were significantly less than in PC9 cells (Fig. 5). In addition, when the three NSCLC cell lines were exposed to docetaxel at its $\mathrm{IC}_{50}$-value dose for $72 \mathrm{~h}$, the levels of $\mathrm{p}$-EGFR 

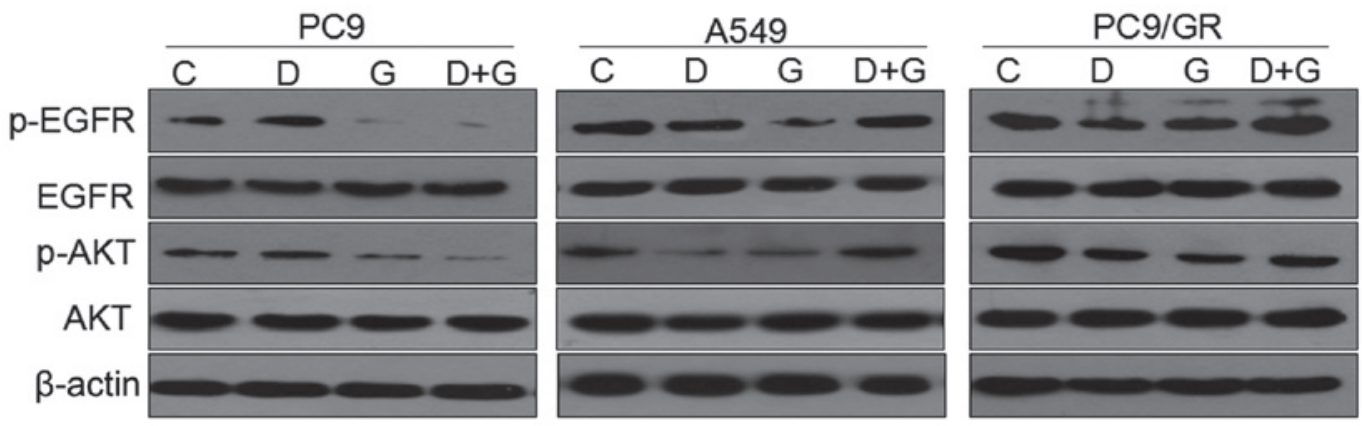

Figure 5. The effects of gefitinib and docetaxel alone and concurrent exposure for $72 \mathrm{~h}$ at their IC $\mathrm{I}_{50}$ concentration. Proteins of EGFR and its downstream signaling pathways were analyzed subsequent to treatment by western blot analysis with corresponding antibodies. The expression levels of the proteins were detected in PC9, A549 and PC9/GR cells. $\beta$-actin was used as loading control. C, D, G and D+G refer to control, docetaxel, gefitinib and concurrent administration, respectively. EGFR, epidermal growth factor receptor.

and p-AKT were increased in PC9 cells, while in A549 and PC9/GR cells, docetaxel decreased the levels of p-EGFR and p-AKT compared with those of unexposed cells.

Additionally, when the PC9 cells were exposed to a combination of docetaxel and gefitinib for $72 \mathrm{~h}$, the levels of $\mathrm{p}$-EGFR and p-AKT were significantly decreased compared with their levels in the control, whereas, concurrent administration of the two drugs increased the levels of p-AKT and p-EGFR in the A549 and PC9/GR cells. However, compared with the control, there was no significant alteration in the total EGFR and AKT expression (Fig. 5).

\section{Discussion}

EGFR-targeted anticancer agents, including gefitinib and erlotinib, have improved the survival rates in patients whose tumors harbor activating mutations within the EGFR gene $(3,4)$. However, the presence of primary or acquired resistance to EGFR-TKIs has limited the effects of these targeted drugs. Thus, the development of novel treatment strategies for patients with NSCLC is an urgent clinical objective.

Previous in vitro and in vivo studies suggest that EGFR-TKIs enhance the anticancer effects of specific conventional cytotoxic drugs, which may lead to less toxic and more effective cancer treatment options (20-22).

However, the concomitant use of EGRF-TKIs with cytotoxic chemotherapy in four clinical randomized phase III trials of INTACT-1, INTACT-2, TALENT and TRIBUTE showed no survival improvement over chemotherapy alone in patients with advanced NSCLC $(15,16,23,24)$. The unexpected negative results may have been explained by either the lack of patient selection or antagonism between EGFR-TKIs and chemotherapeutic agents $(17,18)$.

The present study was performed in EGFR-TKI-sensitive PC9 (EGFR mutant/wild-type K-Ras), EGFR-TKI-primary resistant A549 (wild-type EGFR/mutant K-Ras) and EGFR-TKI acquired-resistant PC9/GR human lung cancer cell lines to investigate the antiproliferative effects of gefitinib and docetaxel as single agents or in combined treatment.

Docetaxel and gefitinib exhibited dose-dependent antiproliferative effects when used as single agents to treat A549, PC9 and PC9/GR lung cancer cells. However, the $\mathrm{IC}_{50}$ values of gefitinib in A549 and PC9/GR cell lines were higher compared with gefitinib-sensitive PC9 cells. Notably, synergism was only observed in the PC9 cells when gefitinib was combined with docetaxel. By contrast, in the EGFR-TKI-resistant A549 and PC9/GR cells, antagonistic interactions were observed upon concomitant administration. The antiproliferative effects in EGFR-TKIs-resistant cell lines were consistent with those observed in a previous study (25).

A similar antitumor effect of combination therapy on cell apoptosis was also observed in the three NSCLC cell lines. Gefitinib induced minimal apoptosis in A549 and PC9/GR cells, whereas PC9 cells were more sensitive. Docetaxel alone induced marked apoptosis in all three cells examined. Notably, the combination of gefitinib and docetaxel exhibited superior rates of apoptosis only in the PC9 cell line with an EGFR mutation.

The mechanisms of the synergistic and antagonistic effects in different cell lines may be attributed to cell cycle distributions. In the current study, gefitinib arrested cells at the G0/G1 phase and docetaxel caused mainly S or G2/M phase accumulation in three cell lines. In the PC9 cells, when exposed to a combination of docetaxel and gefitinib, a cell cycle arrest pattern was observed that was similar to that resulting from treatment with docetaxel administered alone. However, in the A549 and PC9/GR cells, the concurrent administration of docetaxel and gefitinib resulted in alterations in the cell cycle phase distribution and overlapping effects from the two agents. Other studies also suggested that concurrent administration of EGFR-TKIs and chemotherapy resulted in an antagonistic interaction resulting from mutual cell cycle interference $(26,27)$.

The differences in the antiproliferative effects of gefitinib combined with docetaxel may also result from their effects on growth signaling pathways. AKT is an important downstream target of the EGFR pathway and is known to inhibit apoptosis in several ways (28). The results from the current study showed that in the PC9 cells, which are highly sensitive to gefitinib, there was a marked inhibition of p-AKT and p-EGFR following treatment with gefitinib. By contrast, in the EGFR wild-type A549 and gefitinib-acquired-resistant PC9/GR cell lines, there was no significant inhibition of p-EGFR and p-AKT following treatment with gefitinib. In the PC9 EGFR mutant cells, an increase in p-EGFR and p-AKT was observed following docetaxel treatment. By contrast, in the EGFR-TKI-resistant A549 and PC9/GR cells, docetaxel exposure did not result in increased EGFR or AKT phosphorylation. The results were inconsistent with those of previous studies, which reported that docetaxel increased the levels of phosphorylated AKT 
in EGFR-TKI-resistant cell lines (29). The conflicting results may be in part due to the different heritage characteristics of the cell lines and a lower concentration of docetaxel.

When gefitinib was combined with docetaxel, a significant decrease in p-AKT and p-EGFR levels was observed in the PC9 cells, as compared with the control. However, in the A549 EGFR-TKI-primary resistant cells and PC9/GR EGFR-TKI-acquired resistant cells, the levels of p-EGFR and p-AKT increased when gefitinib and docetaxel were applied together. These observations of p-EGFR and p-AKT in NSCLC cells indicate that the interactions between gefitinib and docetaxel may be associated with the effect of docetaxel on EGFR phosphorylation and AKT phosphorylation. Similar to the current results, previous studies reported that EGFR phosphorylation levels following chemotherapy determine the response to combined gefitinib/chemotherapy treatment in NSCLC cells $(30,31)$.

In conclusion, the present study demonstrated that docetaxel in combination with gefitinib, resulted in differences in inhibition of tumor cell proliferation and rates of apoptosis in three NSCLC cell lines in vitro. The observation of synergistic and antagonistic effects between gefitinib and docetaxel in the three NSCLC cell lines may indicate that coadministration of gefitinib and docetaxel may be beneficial to NSCLC patients with EGFR mutant tumors. However, to be able to generalize, confirmation of this observation in a larger number of NSCLC cell lines is necessary and further studies are required to explore in vivo concurrent administration of gefitinib plus docetaxel in patients with EGFR-TKI-resistant NSCLC.

\section{Acknowledgements}

This study was supported by a grant from the Anhui Provincial Natural Science Research Program of Higher Education Institutions Foundation of China (grant no. KJ2012A157) and supported by the Central Laboratory of the Third Affiliated Hospital of Anhui Medical University. The authors would like to thank Dr Xuchao Zhang for providing the cell lines.

\section{References}

1. Jemal A, Siegel R, Xu J and Ward E: Cancer statistics, 2010. CA Cancer J Clin 60: 277-300, 2010.

2. Schiller JH, Harrington D, Belani CP, et al: Comparison of four chemotherapy regimens for advanced non-small-cell lung cancer. N Engl J Med 346: 92-98, 2002.

3. Lynch TJ, Bell DW, Sordella R, et al: Activating mutations in the epidermal growth factor receptor underlying responsiveness of non-small-cell lung cancer to gefitinib. N Engl J Med 350: 2129-2139, 2004.

4. Paez JG, Jänne PA, Lee JC, et al: EGFR mutations in lung cancer: correlation with clinical response to gefitinib therapy. Science 304: 1497-1500, 2004

5. Pao W, Wang TY, Riely GJ, et al: KRAS mutations and primary resistance of lung adenocarcinomas to gefitinib or erlotinib. PLoS Med 2: e17, 2005.

6. Jackman D, Pao W, Riely GJ, et al: Clinical definition of acquired resistance to epidermal growth factor receptor tyrosine kinase inhibitors in non-small-cell lung cancer. J Clin Oncol 28 357-360, 2010.

7. Parkin DM, Bray F, Ferlay J and Pisani P: Global cancer statistics, 2002. CA Cancer J Clin 55: 74-108, 2005.

8. Gloeckler Ries LA, Reichman ME, Lewis DR, Hankey BF and Edwards BK: Cancer survival and incidence from the Surveillance, Epidemiology, and End Results (SEER) program. Oncologist 8: 541-552, 2003.
9. Schiff PB, Fant J and Horwitz SB: Promotion of microtubule assembly in vitro by taxol. Nature 277: 665-667, 1979.

10. Gligorov J and Lotz JP: Preclinical pharmacology of the taxanes: implications of the differences. Oncologist 9 (Suppl 2): 3-8, 2004.

11. Fukuoka M, Yano S, Giaccone G, et al: Multi-institutional randomized phase II trial of gefitinib for previously treated patients with advanced non-small-cell lung cancer (The IDEAL 1 Trial) [corrected]. J Clin Oncol 21: 2237-2246, 2003.

12. Kris MG, Natale RB, Herbst RS, et al: Efficacy of gefitinib, an inhibitor of the epidermal growth factor receptor tyrosine kinase, in symptomatic patients with non-small cell lung cancer: a randomized trial. JAMA 290: 2149-2158, 2003.

13. Ciardiello F, Caputo R, Bianco R, et al: Antitumor effect and potentiation of cytotoxic drugs activity in human cancer cells by ZD-1839 (Iressa), an epidermal growth factor receptor-selective tyrosine kinase inhibitor. Clin Cancer Res 6: 2053-2063, 2000.

14. Sirotnak FM, Zakowski MF, Miller VA, Scher HI and Kris MG: Efficacy of cytotoxic agents against human tumor xeno-grafts is markedly enhanced by coadministration of ZD1839 (Iressa), an inhibitor of EGFR tyrosine kinase. Clin Cancer Res 6: 4885-4892, 2000.

15. Herbst RS, Giaccone G, Schiller JH, et al: Gefitinib in combination with paclitaxel and carboplatin in advanced non-small-cell lung cancer: a phase III trial - INTACT 2. J Clin Oncol 22: 785-794, 2004.

16. Giaccone G, Herbst RS, Manegold C, et al: Gefitinib in combination with gemcitabine and cisplatin in advanced non-small-cell lung cancer: a phase III trial - INTACT 1. J Clin Oncol 22: 777-784, 2004.

17. Gandara D, Narayan S, Lara PN Jr, et al: Integration of novel therapeutics into combined modality therapy of locally advanced non-small cell lung cancer. Clin Cancer Res 11: 5057s-5062s, 2005.

18. Gandara DR and Gumerlock PH: Epidermal growth factor receptor tyrosine kinase inhibitors plus chemotherapy: case closed or is the jury still out? J Clin Oncol 23: 5856-5858, 2005.

19. Chou TC and Talalay P: Quantitative analysis of dose-effect relationships: the combined effects of multiple drugs or enzyme inhibitors. Adv Enzyme Regul 22: 27-55, 1984.

20. Magné N, Fischel JL, Dubreuil A, et al: Sequence-dependent effects of ZD1839 ('Iressa') in combination with cytotoxic treatment in human head and neck cancer. Br J Cancer 86: 819-827, 2002.

21. Xu JM, Azzariti A, Colucci G and Paradiso A: The effect of gefitinib (Iressa, ZD1839) in combination with oxaliplatin is schedule-dependent in colon cancer cell lines. Cancer Chemother Pharmacol 52: 442-448, 2003.

22. Higgins B, Kolinsky K, Smith M, et al: Antitumor activity of erlotinib (OSI-774, Tarceva) alone or in combination in human non-small cell lung cancer tumor xenograft models. Anticancer Drugs 15: 503-512, 2004.

23. Herbst RS, Prager D, Hermann R, et al: TRIBUTE: A phase III trial of erlotinib hydrochloride (OSI-774) combined with carboplatin and paclitaxel chemotherapy in advanced non-small-cell lung cancer. J Clin Oncol 23: 5892-5929, 2005.

24. Gatzemeier U, Pluzanska A, Szczesna A, et al: Phase III study of erlotinib in combination with cisplatin and gemcitabine in advanced non-small-cell lung cancer: the Tarceva Lung Cancer Investigation Trial. J Clin Oncol 25: 1545-1552, 2007.

25. Cheng H, An SJ, Zhang XC, et al: In vitro sequence-dependent synergism between paclitaxel and gefitinib in human lung cancer cell lines. Cancer Chemother Pharmacol 67: 637-646, 2011.

26. Davies AM, Ho C, Lara PN Jr, Mack P, Gumerlock PH and Gandara DR: Pharmacodynamic separation of epidermal growth factor receptor tyrosine kinase inhibitors and chemotherapy in non-small-cell lung cancer. Clin Lung Cancer 7: 385-388, 2006.

27. Li T, Ling YH, Goldman ID and Perez-Soler R: Schedule-dependent cytotoxic synergism of pemetrexed and erlotinib in human non-small cell lung cancer cells. Clin Cancer Res 13: 3413-3422, 2007.

28. Cross TG, Scheel-Toellner D, Henriquez NV, Deacon E, Salmon M and Lord JM: Serine/threonine protein kinases and apoptosis. Exp Cell Res 256: 34-41, 2000.

29. Pan F, Tian J, Zhang X, et al: Synergistic interaction between sunitinib and docetaxel is sequence dependent in human non-small lung cancer with EGFR TKIs-resistant mutation. J Cancer Res Clin Oncol 137: 1397-1408, 2011.

30. Van Schaeybroeck S, Karaiskou-McCaul A, Kelly D, et al: Epidermal growth factor receptor activity determines response of colorectal cancer cells to gefitinib alone and in combination with chemotherapy. Clin Cancer Res 11: 7480-7489, 2005.

31. VanSchaeybroeck S,Kyula J,Kelly DM,etal:Chemotherapy-induced epidermal growth factor receptor activation determines response to combined gefitinib/chemotherapy treatment in non-small cell lung cancer cells. Mol Cancer Ther 5: 1154-1165, 2006. 